\title{
Environment of $\mathrm{Er}$ in a-Si:H: Co-Sputtering Versus Ion Implantation
}

\author{
Cínthia Piamonteze, Leandro R. Tessler, \\ Instituto de Fisica "Gleb Wataghin", UNICAMP, C. P. 6165 , \\ 13083-970, Campinas, SP, Brazil \\ M. C. Martins Alves, and H. Tolentino \\ Laboratório Nacional de Luz Síncrotron, \\ C. P. 6192, 13083-970, Campinas, SP, Brazil
}

Received February 6, 1999

\begin{abstract}
We report a comparative Extended X-Ray Fine Structure (EXAFS) study of Er in $a$-Si:H prepared by Er implantation in $a$-Si:H and by co-sputtering undergoing the same cumulative annealing processes. It was found that the Er environment in as-implanted samples is formed by Si atoms, which are replaced by oxygen under annealing. In the co-sputtered samples, the initial low coordination oxygen environment evolves under thermal treatment to an $\mathrm{Er}_{2} \mathrm{O}_{3}$-like neighborhood.
\end{abstract}

\section{Introduction}

The luminescence of triply ionized erbium $\left(\mathrm{Er}^{3+}\right)$ in semiconductors has motivated many studies of this subject because of potential photonic applications. The $\mathrm{Er}^{3+}$ luminescence arises from atomic-like electronic transitions within its incomplete $4 f$ shell. An emission at $\sim 1.54 \mu \mathrm{m}$ comes from the transition between the first excited and the fundamental state $\left({ }^{4} I_{13 / 2} \rightarrow{ }^{4} I_{15 / 2}\right)$ of the ion $\mathrm{Er}^{3+}$. Since this transition does not involve bonding electrons and the $4 f$ shell is shielded by the outer $5 s^{2} 5 d^{6}$ shells, its energy levels are relatively insensitive to the Er chemical neighborhood, i. e., depend very weakly on the host. The transition probability, however, depends on the symmetry of the $\mathrm{Er}^{3+}$ site in the host. Intra-4f transitions are electric dipole forbidden and only in a symmetry breaking environment the parity of the wavefunction is modified making the transition at least partially allowed. The wavelength of the photon associated to this luminescence $(1.54 \mu \mathrm{m})$ is of particular interest because it corresponds to the window of minimum absorption loss in silicon based optical fibers. Silicon has been one of the most studied hosts for erbium because of the possibility of photonic-electronic integration. In silicon, the luminescence intensity has been found to be strongly enhanced by the presence of impurities like oxygen. Moreover, the $\mathrm{Er}^{3+}$ luminescence intensity is higher in hydrogenated amorphous silicon (a-Si:H) than in pure crystalline silicon (c-Si) [1].

The chemical neighborhood of erbium has been investigated in crystalline and amorphous silicon by Extended X-Ray Absorption Fine Structure (EXAFS). It has been found [2] that efficient $\mathrm{Er}^{3+}$ luminescence in Czochralski silicon (Cz-Si) is associated to an Er local environment very similar to that of $\mathrm{Er}_{2} \mathrm{O}_{3}$. In $\mathrm{Er}_{2} \mathrm{O}_{3}$ Er sits in a 6-fold coordinated cage, which according to [2] provides a non-centrosymmetric environment that allows the optical transition, in contrast to the highly symmetric 12-fold Si cage found in float-zone silicon (FZ-Si), where the Er centers are optically inactive. An investigation [3] of annealing effects on crystalline Si coimplanted with Er and O, it has been found that the chemical environment of Er evolves from a 6-fold silicon coordination shell to a 5 -fold oxygen coordination shell upon thermal annealing. In samples of non-annealed, co-sputtered Er doped a-Si:H and a- $\mathrm{SiO}_{x}: \mathrm{H}$ with controlled oxygen contents [4] we have found that $\mathrm{Er}$ is coordinated to oxygen, like in annealed $\mathrm{Cz}-\mathrm{Si}$. However, the maximum average $\mathrm{O}$ coordination is 3.6 providing for Er a local environment of lower symmetry and thus enhancing the probability of the luminescent transition.

In this paper we present a comparative EXAFS 
study between Er in a-Si:H prepared by co-sputtering and by Er implantation in sputtered a-Si:H. The evolution of the Er chemical environment with thermal annealing in samples prepared by the two procedures undergoing the same cumulative annealing steps is found to be significantly different.

\section{Experiment}

An erbium doped a-Si:H sample was prepared in a conventional rf sputtering system following a procedure described elsewhere [5]. The oxygen to silicon concentration $[\mathrm{O}] /[\mathrm{Si}]$ measured by Rutherford Backscattering spectroscopy (RBS) is $~ 7.6$ at. \%, and $[\mathrm{Er}] /[\mathrm{Si}]$ is $\sim 0.6$ at. \%. This high oxygen concentration is due to preparation under sputtering conditions (low bias) leading to columnar a-Si:H, which optimize the $\mathrm{Er}^{3+}$ photoluminescence in samples not intentionally oxygenated [6]. The Er implanted a-Si:H sample was prepared under sputtering conditions optimized for good quality a-Si:H deposition. An Er concentration of $10^{16} \mathrm{~cm}^{-2}$, which corresponds to $[\mathrm{Er}] /[\mathrm{Si}] \sim 0.2$ at.\%, was obtained by ion implantation at IF-UFRGS, Porto Alegre, Brazil. The residual oxygen concentration in this sample is $[\mathrm{O}] /[\mathrm{Si}]$ $\sim 0.2$ at.\%, comparable to the Er concentration.

Erbium $\mathrm{L}_{I I I^{-}}$edge EXAFS was measured with secondary electron total yield detection at the XAFS beamline [7] of the Laboratório Nacional de Luz Síncrotron (LNLS) in Campinas, Brazil. The samples were at room temperature during the measurements. Samples of bulk $\mathrm{Er}_{2} \mathrm{O}_{3}$ and a thin film of $\mathrm{ErSi}_{2}$ were used as reference standards for determining coordination numbers and bond lengths. EXAFS data were processed following the recommendations of the International Committee [8].

The samples were annealed ex-situ in a nitrogen atmosphere at constant temperatures by 20 minutes steps. The temperatures used in the cumulative annealing varied between $200^{\circ} \mathrm{C}$ and $1100^{\circ} \mathrm{C}$ in $\sim 100^{\circ} \mathrm{C}$ steps.

\section{Results}

In figure 1 we show the pseudo-radial distribution functions ( $p$-RDF) obtained from the Fourier transforms of the EXAFS oscillations $\chi(\mathrm{k}) \cdot \mathrm{k}^{2}$. Curves for the two samples, as deposited and annealed at $1100^{\circ} \mathrm{C}$, which was the maximum temperature used, are represented.
The $p$-RDF for the standard samples are also shown for comparison.

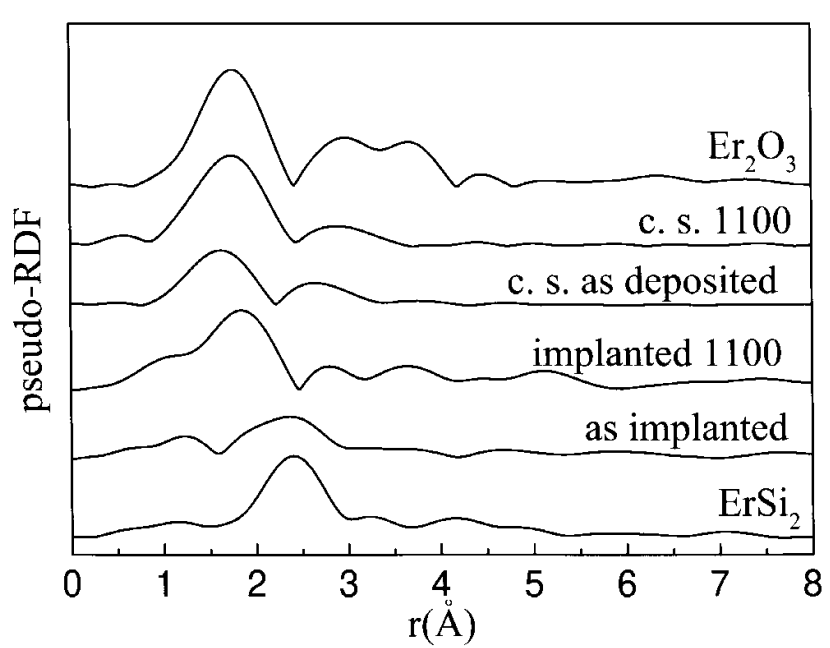

Figure 1. Pseudo-RDF obtained from the $\mathrm{k}^{2}$ weighted Fourier transforms. C. S. indicates the co-sputtered sample. The annealing temperature is also indicated.

In the co-sputtered sample without thermal treatment and annealed the first peak of the $p$-RDF indicates an interatomic separation which is close to the Er-O distance in the standard $\mathrm{Er}_{2} \mathrm{O}_{3}$ sample. This indicates that the erbium first neighbors are oxygen atoms and they do not change under annealing.

The results for the implanted sample are quite different. The first peak of the $p$-RDF for the as implanted sample is close to the Er-Si separation in $\mathrm{ErSi}_{2}$. The $p$-RDF is similar for all the annealing temperatures except for the annealing at $1100^{\circ} \mathrm{C}$, when it becomes $\mathrm{Er}_{2} \mathrm{O}_{3}$ like. We can conclude that erbium is coordinated to silicon up to annealing temperatures of $850^{\circ} \mathrm{C}$. Between $850^{\circ} \mathrm{C}$ and $1100^{\circ} \mathrm{C}$ it becomes coordinated to oxygen.

To obtain the average atomic separation, the average coordinations and the Debye-Waller factor relative to the first neighbor shell we calculated the backtransforms of the isolated first peaks of the $p$-RDF and compared them with simulations using the amplitudes and phases obtained from the standard samples. The $\mathrm{Er}_{2} \mathrm{O}_{3}$ and $\mathrm{ErSi}_{2}$ standards were used for oxygen and silicon neighbor shell respectively.

\section{Discussion}

Let us first discuss the co-sputtered sample. Under annealing, the erbium chemical environment evolves to- 
wards that of $\mathrm{Er}_{2} \mathrm{O}_{3}$, where erbium is coordinated with 6 oxygen atoms and the Er-O distance is $2.26 \AA$ [2]. This can be seen in figures 2 and 3 where the average ErO distance and erbium coordination are represented versus the annealing temperature. Both first neighbor separation and coordination increase with annealing temperature. The average Er coordination starts at $3.1 \pm 0.1$ as in a-Si:H [4], reaches a maximum value of $6.3 \pm 0.3$ for $850^{\circ} \mathrm{C}$ annealing and remains at this value. The maximum Er-O distance of $2.27 \pm 0.01 \AA$ is reached for $1100^{\circ} \mathrm{C}$ annealing.

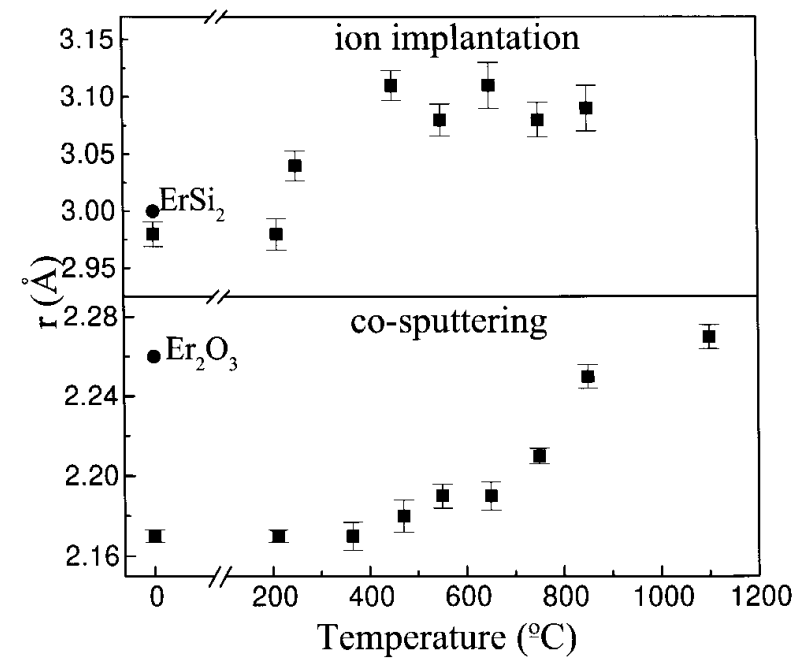

Figure 2. Er first neighbor separation in the implanted and co-sputtered samples. The values for $\mathrm{ErSi}_{2}$ and $\mathrm{Er}_{2} \mathrm{O}_{3}$ standards are indicated.

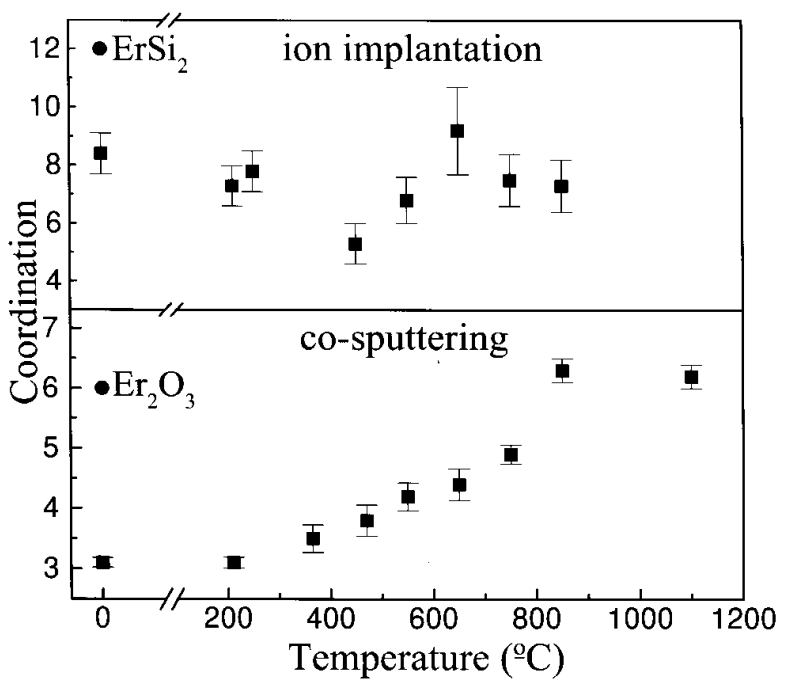

Figure 3. Er coordination in the implanted and co-sputtered samples. The values for $\mathrm{ErSi}_{2}$ and $\mathrm{Er}_{2} \mathrm{O}_{3}$ standards are indicated.

In contrast, the behavior of the average Er coordination and first neighbor separation with annealing is very different for the implanted sample, as shown in figures 2 and 3. The Er-Si separation for the as deposited sample is $2.98 \pm 0.01 \AA$, very close to the Er-Si distance in the standard $\mathrm{ErSi}_{2}$ which is $3.00 \AA$. This distance starts to increase for annealing at $250^{\circ} \mathrm{C}$ and reaches a maximum at $450^{\circ} \mathrm{C}(3.11 \pm 0.02 \AA)$. At higher annealing temperatures the Er-Si distance remains constant. The Er coordination is approximately constant except for $450^{\circ} \mathrm{C}$ annealed sample, which shows a pronounced decrease. The sample looked opaque after $550^{\circ} \mathrm{C}$ annealing, indicating the onset of Si crystallization.

The Er first neighbor separation and coordination for $1100^{\circ} \mathrm{C}$ annealing are not shown in the figures because at this temperature the Er first neighbor becomes oxygen. The value found for the Er-O separation in the implanted sample annealed at $1100^{\circ} \mathrm{C}$ is $2.28 \pm 0.02$ and the coordination is $6.5 \pm 0.6$, indicating that the Er local environment in this sample is very similar to that in $\mathrm{Er}_{2} \mathrm{O}_{3}$.

\section{Conclusions}

In the sample prepared by co-sputtering, Er is incorporated in such a way that it finds a configuration that minimizes the local free energy of the amorphous system. In this configuration, Er is surrounded in average by 3 oxygen atoms with an interatomic distance much smaller than in $\mathrm{Er}_{2} \mathrm{O}_{3}$ [4]. In the sample prepared by ion implantation the violent Er incorporation does not allow system relaxation. Since the oxygen concentration is small, Er is coordinated to silicon.

The expected effect of the annealing process is to let the system evolve to a lower free energy configuration. In the co-sputtered sample there is enough oxygen to satisfy the $\mathrm{Er} 6$-fold coordination like in $\mathrm{Er}_{2} \mathrm{O}_{3}$. Thus the Er chemical environment evolves to that found in this crystal. In the implanted sample, $\mathrm{Er}_{2} \mathrm{O}_{3}$-like environment was only detected after annealing at $1100^{\circ} \mathrm{C}$. Observation of the sample in a microscope after this annealing step showed that parts of the $a-\mathrm{Si}: \mathrm{H}$ film had been ablated. This probably opens easy oxidation ways allowing for the formation of $\mathrm{Er}_{2} \mathrm{O}_{3}$ - like environments.

For the implanted sample both the Er-Si distance and the Er coordination have an anomalous behavior at $450^{\circ} \mathrm{C}$. We propose the following simple model: with 
the thermal treatment the host material relaxes by creating microvoids around the Er atoms. Consequently the Er coordination decreases and the Er-Si separation increases. For higher annealing temperatures silicon crystal nucleation starts causing an increase in the Er coordination whereas the Er-Si distance keeps approximately constant. Therefore, the local symmetry decreases with annealing temperature until crystallization starts. This may be the reason why the maximum $\mathrm{Er}^{3+}$ photoluminescence is found for annealing temperatures below $500^{\circ} \mathrm{C}[9]$.

In summary, we have found that not only the $\mathrm{Er}$ chemical environment is different in the two as prepared samples but also that the evolution of this environment upon thermal treatment differ. The structural changes in the implanted sample lead to a more asymmetrical Er environment up to $450^{\circ} \mathrm{C}$ annealing. This enhances the luminescence intensity. The Er chemical neighborhood in the sample prepared by co-sputtering evolves to that of $\mathrm{Er}_{2} \mathrm{O}_{3}$.

\section{Acknowledgments}

The authors are indebted to A. C. Iñiguez for sample preparation, to L. Amaral for ion implantation and to the LNLS staff for providing the photons. This work was partially supported by FAPESP, CNPq and PRONEX "Fotônica em Telecomunicações".

\section{References}

[1] M. S. Bresler, O. B. Gusev, V. Kh. Kudoyarova, A. N. Kuznetsov, P. E. Pak, E. I. Terukov, I. N. Yassievich, B. P. Zakharchenya, W. Fuhs and A. Sturm, Appl. Phys. Lett. 67, 3599 (1995).

[2] D. L. Adler, D. C. Jacobson, D. J. Eaglesham, M. A. Marcus, J. L. Benton, J. M. Poate, and P. H. Citrin, Appl. Phys. Lett. 61, 2181 (1992).

[3] A. Terrasi, G. Franzò, and S. Coffa, F. Priolo, F. D 'Acapito, S. Mobilio, Appl. Phys. Lett. 70, 1712 (1997).

[4] C. Piamonteze, A. C. Iñiguez, L. R. Tessler, M. C. Martins Alves and H. Tolentino, Phys. Rev. Lett. 81, 4652 (1998).

[5] L. R. Tessler and A. C. Iñiguez, in Amorphous Silicon Technology -1998, (MRS Symp. Proc. 507, Pittsburgh, PA 1998), in press.

[6] L. R. Tessler and A. R. Zanatta, J. Non-Cryst. Sol. 227-230, 399 (1998).

[7] H. Tolentino, J. C. Cezar, D. Z. Cruz, V. CompagnonCailhol, E. Tamura, M. C. Martins Alves, J. Synchrotron Rad. 5, 521 (1998).

[8] F. W. Lytle, D. E. Sayers, and E. A. Stern, Physica (Amsterdam) 158B, 701 (1989).

[9] J. H. Shin, R. Serna, G. N. van den hoven, A. Polman, W. G. J. H. M. van Sark and A. M. Vredenberg, Appl. Phys. Lett. 68, 997 (1996). 